\title{
QUASI-MOLECULE MODEL OF THE SPECTRA OF MOLECULES IN POLAR SOLUTION IN CASE OF TIME DEPENDENT FLUORESCENCE SHIFTS
}

\author{
A. Bączyński, T. Marszalek, D. Radomska, P. Targowski \\ AND B. ZIETEK
}

Institute of Physics, N. Copernicus University, Grudziądzka 5/7, 87-100 Toruń, Poland

(Received May 18, 1992)

\begin{abstract}
Analytical expressions of profiles of absorption and instantancous fuorescence spectra of dye molecules in polar solvents are given. It is shown what parameters can be gained from electronic spectra of dye solutions. The new procedure of interpretation of transient fluorescence spectra is demonstrated in the case of the spectra of nile blue in alcohol.
\end{abstract}

PACS numbers: $33.50 . \mathrm{Dq}, 78.55 . \mathrm{Bq}$

\section{Introduction}

Absorption and emission of a given molecular system are characterized by their specific spectral distributions. Great effort was undertaken to get spectral profiles of liquid dye solutions in analytical forms. One of the infallible methods for a spectral profile description is the momentum method. An observed spectral distribution, independently on a theoretical specification of the system, is represented by the set of moments expressing the maximum positions, half-widths and asymetries of the spectrum [1]. It appears that in most cases of structureless bands the first three moments suffice for the description of their profiles.

Another spectral distribution, accurately reproducing electronic band profiles is the log-normal distribution [2,3]. This distribution is also a three parameter distribution similar to those, based on the configurational model [4]. Many attempts were undertaken for the adaptations of Gaussian distributions and their superpositions to represent the molecular spectra. Unfortunately molecular electronic spectra never appear as Gaussian. The superposition of Gaussian curves needs more than three parameters, besides it suggests scveral numbers of transitions, which may not be present. It is a point why does the log-normal distribution fit so well to the description of so many molecular systems? 
A very important problem is to gain physical interpretation for the parameters, appearing in the spectral distributions used. This problem, independent of many attempts, does not seem to be solved yet. The aim of this paper is to show what knowledge can be gained from molecular electronic spectra of complex molecules in polar solvents. It is believed that the solvatochromic effect, the characteristic interaction between solute and solvent molecules is to a large extent responsible for the appearance of spectral profiles [5]. It appears that solute molecules with a dipole moment in polar solvents interact so strongly that a quasi-molecule rather than an isolated molecule has to be considered. A quasi-molecule concept assumes a physical bond between solute and the surrounding solvent molecules [6].

\section{Description of the model}

Before light absorption takes place, the solvent molecules are in equilibrium with the solute molecules in their ground states and the quasi-molecules are in an equilibrated ground state. The excitation causes an abrupt change of the charge distribution in the solute molecule, but the configuration of solvent molecules remains unchanged. Immediately after excitation the quasi-molecule is initially in an highly nonequilibrated excited state which is referred to as a Franck-Condon state.

The thermalization process of the FC state consists of a very fast vibrational redistribution, of a cooling effect to reach the temperature of the surroundings [7] and, in a longer time scale, of the dynamic solvatochromic effect [8]. It means that immediately after excitation relaxation processes compete with simultaneous deexcitation. The manifestation of all these processes is the dynamic Stokes shift $[9,10]$ which can be observed directly in time resolved fluorescence spectra [11-15].

When the decay time of fluorescence is much longer than the time constant of reorientation of the solvent molecules, the stationary fluorescence spectrum may be thought of as a result of an electronic transition from the equilibrated excited quasi-molecule (different from the equilibrated ground state of the quasi-molecule). Though the equilibrium state in liquids is rcached very soon, in some tenths of picoseconds [16], the result of the equilibration processes can be observed even in steady state fluorescence spectra [17].

The quasi-molecule consists of a solute molecule and its nearest surroundings. In the case of strong solute-solvent interaction the wave function of such a system in an adiabatic approximation is scparated into the electronic part and the part depending on nuclear coordinates of the quasi-molecule. Because of the great number of degrees of freedom, in each electronic state there exists a great number of sublevels which, to a good approximation, can be treated as a continuum. It appears that the potential energy of a system with so many degrees of freedom may be approximated to a function of a single variable. This variable however by no means may be treated as a definite coordinate [18].

The expression of the potential energy as a function of one variable only, in harmonic approximation, with the admission of different potentials for different electronic states leads to analytical expressions for absorption and emission spectra in unified manner [6]. The derivation is based on the classical formulation of the 
Franck-Condon principle, with the assumption of continuum of sublevels in initial and final states with Boltzmann population distribution in the former.

Oscillations of the quasi-molecule in an external field of a solvent are controlled by potential energy that can be expressed in terms of displacements of configurational coordinates from their equilibrium positions. For small displacements a harmonic approximation is justified; a coordinate system is chosen such that the equilibrium position of the quasi-molecule in the ground state is at $Q=0$, and in the excited state at $Q=\left(Q_{0}\right)_{t}$. The subscript $t$ indicates that the equilibrium position in the excited state is not determined once for ever, but is a dynamical parameter. Its value changes moderately during the evolution of the quasi-molecule. Because this value is not known, it is convenient to express the potential energies by $y=Q /\left(Q_{0}\right)_{t}$. In these coordinates the potential energy of the ground and the first excited states at the moment of absorption, at $t=0$, is given by

$$
\begin{aligned}
& W_{\mathrm{g}}^{0}(y)=k_{\mathrm{g}}^{0}\left(Q_{0}\right)_{t=0}^{2} y^{2}, \\
& W_{\mathrm{e}}(y)=\left(k_{\mathrm{e}}\right)_{t=0}\left(Q_{0}\right)_{t=0}^{2}(y-1)^{2}+b_{t=0},
\end{aligned}
$$

where $k_{\mathrm{g}}^{0}$ is the force constant in the equilibrated ground state and $\left(k_{\mathrm{e}}\right)_{t=0}$ is the force constant of the quasi-molecule in its excited state just after absorption. The $b$ parameter, being the energy of pure electronic transition, changes with time too.

It is easy to show that putling $c_{a}=k_{\mathrm{g}}^{0}\left(Q_{0}\right)_{t=0}^{2}, b_{a}=b_{t=0}$ and $m=\left(k_{\mathrm{e}}\right)_{t=0} / k_{\mathrm{g}}^{0}$, the main contribution to the absorption given in the preceding paper [6] is

$$
A(\varepsilon)=a(\varepsilon) / a\left(\varepsilon_{m}\right)
$$

with

$$
a(\varepsilon)=\frac{\varepsilon}{M(\varepsilon)} \exp \left\{\frac{-c_{a}[m-M(\varepsilon)]^{2}}{k T(m-1)^{2}}\right\}
$$

and

$$
\varepsilon>b_{a}-m c_{a} /(m-1),
$$

where

$$
M(\varepsilon)=\sqrt{m+(m-1)\left(\varepsilon-b_{a}\right) / c_{a}} .
$$

In this formula $\varepsilon$ is the frequency of the transition, $k$ - the Boltzmann constant, $T$ - the temperature and the maximum of absorption is at $\varepsilon_{m}$.

Because of the relaxation processes the parameters $k_{\mathrm{e}}, Q_{0}$ and $b_{t}$ are time dependent. No matter at what instant of time the emission takes place, the ground state produced will be out of equilibrium and only relaxation processes in the ground state will lead the system to the initial state of equilibrium, like before the promotion of the system to the excited state. The potential energy of the initial and final states of fluorescence, using the same convention, will be given by equations

$$
\begin{aligned}
& W_{\mathrm{e}}(y)=\left(k_{\mathrm{e}}\right)_{t}\left(Q_{0}\right)_{t}^{2}(y-1)^{2}+b_{t}, \\
& W_{\mathrm{g}}(y)=\left(k_{\mathrm{g}}\right)_{t}\left(Q_{0}\right)_{t}^{2} y^{2} .
\end{aligned}
$$


With the same arguments like those which were used to derive the analytical expression (2), the time-dependent profile of the fluorescence spectrum is given by

$$
F(\varepsilon)=f(\varepsilon) / f\left(\varepsilon_{m}\right)
$$

with

and

$$
f(\varepsilon)=\frac{\varepsilon^{3}}{N(\varepsilon)} \exp \left\{\frac{-c_{f}^{\prime}}{k T(n-1)^{2}}[n-N(\varepsilon)]^{2}\right\}
$$

$$
\varepsilon<b_{f}+n c_{f}^{\prime} /(n-1),
$$

where

$$
N(\varepsilon)=\sqrt{n+(n-1)\left(b_{f}-\varepsilon\right) / c_{f}^{\prime}}
$$

and $c_{f}^{\prime}=\left(k_{\mathrm{e}}\right)_{t}\left(Q_{0}\right)_{t}^{2}, \quad b_{f}=b_{t}, \quad n=\left(k_{\mathrm{g}}\right)_{t} /\left(k_{\mathrm{e}}\right)_{t}$.

The derived analytical expressions for absorption and emission band profiles contain only a limited number of parameters and their physical interpretation is clear. Their numerical values may be gained be fitting the experimentally obtained spectra to the presented theoretical spectral distributions. It can be shown that these distributions are under special conditions similar to the log-normal distribution. The expressions (2) for absorplion and (4) for steady state fluorescence were verified for alcoholic solutions of coumarins and a very good agreement with experiment was proved $[6,17]$. It will be shown that also transient fluorescence spectra fit to the expression (4), where the time dependence of the spectra is due to the time dependence of the parameters.

\section{Transient fluorescence spectra}

Verification of Eq. (4) in describing the steady state fluorescence spectra appeared to be above all expectations positive at least for coumarin solutions [6]. To verify the formula (4) with the emphasized time dependence of parameters, transient fluorescence spectra of alcoholic solutions of nile blue, published by Mokhtari et al. [16] werc analyzed. These spectra were measured by femtosecond optical mixing of fluorescence signal with appropriate ultra fast dye laser pulses [19] in urea crystal.

Fitting the instantaneous fluorescence spectra for different delays between excitations and up-converting gating to the formula (4), the time dependence of parameters in Eq. (4) could be determined and relaxation dynamics analyzed. As an example of the fitting procedure the instantaneous fluorescence spectrum at 1 ps delay time is presented in Fig. 1 together with the calculated one. All other spectra presented in Fig. 2 of the paper [16] are reproduced with the same degree of accuracy. Only on the wings some deviations due to the lack of accuracy can be observed. The obtained values of the parameters are given in Table, where the $\chi^{2}$ parameter is the measure of the accuracy of the fitting procedure used and $n$ is defined in Eq. (4).

It is expected that the system after promotion to the excited state will tend to a state of equilibrium in the excited state. This will be manifested by a decrease 


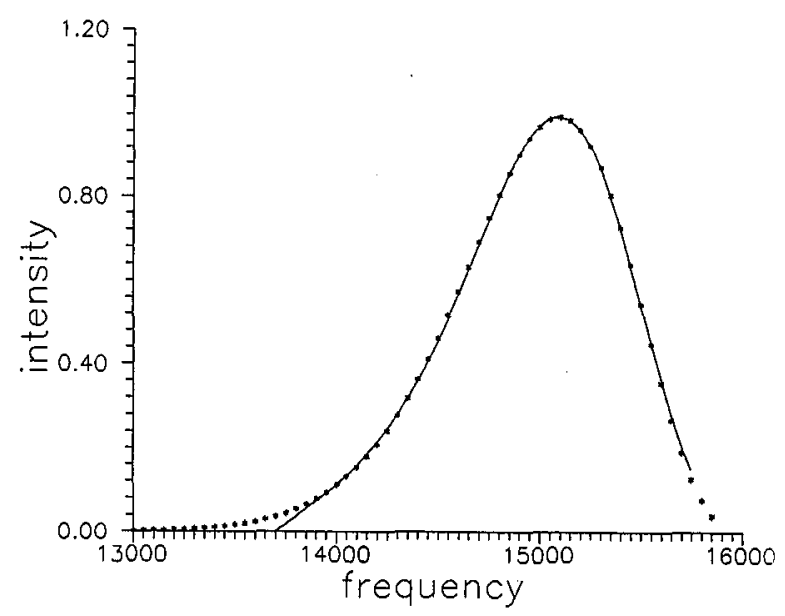

Fig. 1. Instantaneous fluorescence spectrum of nile blue in alcohol for 1 ps time delay after excitation. Solid line: after Mokhtari et al. [16], dots: calculated from Eq. (4).

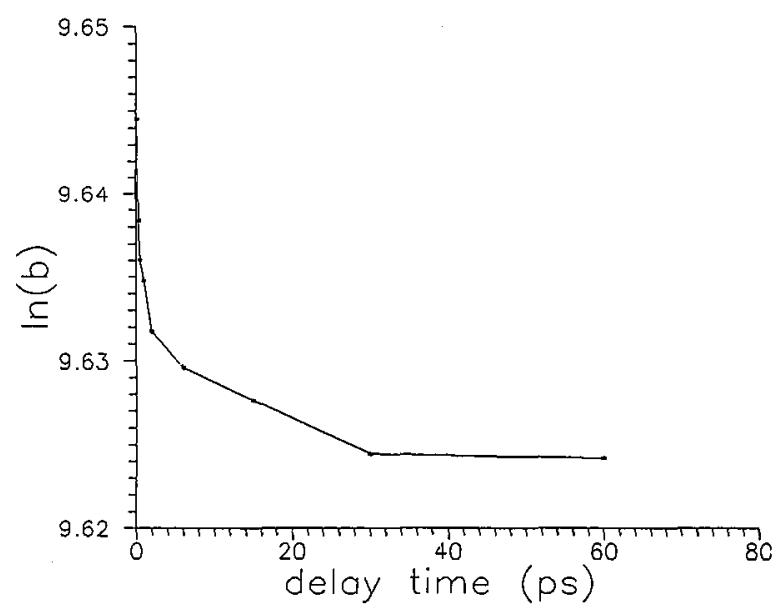

Fig. 2. Time dependence of the pure electronic transition energy. Dots: according to the table.

of the energy of the excited state. The measure of this process is the decrease of the $b_{t}$ parameter, illustrated in Fig. 2 and here, like in the paper [16], the biexponential decay can be noticed.

The time dependence of the product $\left(k_{\mathrm{e}}\right)_{t}\left(Q_{0}\right)_{t}^{2}$ is illustrated in Fig. 3 where stars represent the values obtained by the best fit procedure and the full line is drawn by spline method for better visualization. The subpicosecond part of the curve is obtained by assuming, like for the whole time domain, the temperature value in Eq. (4) being equal to the room temperature. This, as a matter of fact, 
TABLE

Molecular parameters obtained from the best fit of transient fluorescence spectra to the analytical band profile (4).

\begin{tabular}{r|c|c|c|c}
\hline \hline$t[\mathrm{ps}]$ & $b_{t}\left[\mathrm{~cm}^{-1}\right]$ & $\left(k_{\mathrm{e}}\right)_{t}\left(Q_{0}\right)_{t}^{2}\left[\mathrm{~cm}^{-1}\right]$ & $n$ & $\chi^{2}$ \\
\hline 0.15 & $15437 \mp 9$ & $310 \mp 9$ & 1.332 & 0.45 \\
0.30 & $15342 \mp 6$ & $252 \mp 5$ & 1.409 & 0.95 \\
0.50 & $15306 \mp 5$ & $220 \mp 4$ & 1.500 & 0.40 \\
1.0 & $15287 \mp 5$ & $222 \mp 4$ & 1.477 & 0.29 \\
2.0 & $15242 \mp 4$ & $208 \mp 3$ & 1.457 & 0.69 \\
6.0 & $15209 \mp 3$ & $205 \mp 3$ & 1.439 & 0.66 \\
15.0 & $15179 \mp 3$ & $200 \mp 2$ & 1.440 & 0.33 \\
30.0 & $15131 \mp 2$ & $211 \mp 2$ & 1.469 & 0.71 \\
60.0 & $15127 \mp 2$ & $206 \mp 2$ & 1.495 & 0.50
\end{tabular}

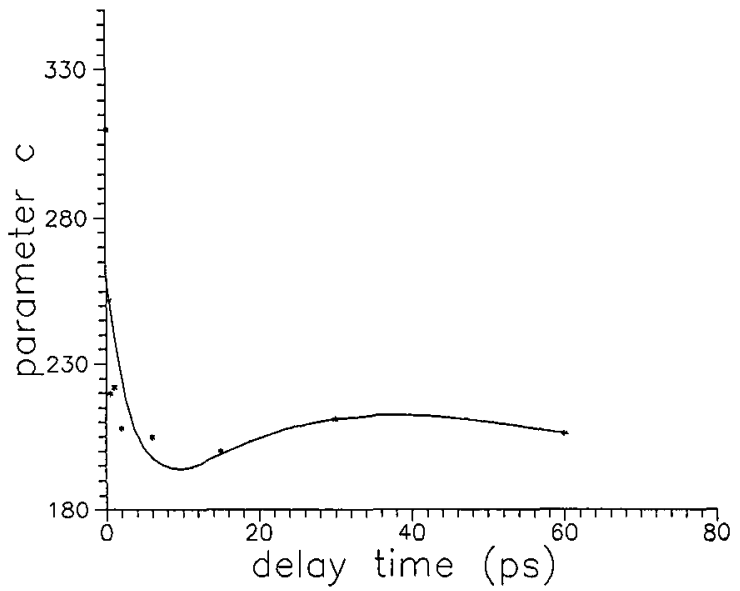

Fig. 3. Time dependence of $c=\left(k_{\mathrm{e}}\right)_{t}\left(Q_{0}\right)_{l}^{2}$. Dots: according to the table, solid line: curve obtained by the spline method.

is not true and therefore this part of the curve serves only for illustration of the vibrational redistribution process. The obtained values of the parameters in this region have no direct physical meaning. After the vibrational relaxation is accomplished, i.e. after approximately 1 ps [20], the proper interpretation of the parameters is assured.

The course of the curve in Fig. 3 gives rise to some speculations. First of all it seems to confirm the earlier suggestion of van der Zwan et al. [10] that the equilibration process of molecular systems may have an inertial component. The other possibility, the assumption of monotonic decrease of the $c$ parameter 
in time after excitation, may be analyzed in terms of separate time dependencies of $\left(k_{\mathrm{e}}\right)_{t}$ and $\left(Q_{0}\right)_{t}$. In that case it would be reasonable to assume that the main contribution to the observed behaviour would be due to the monotonic decrease of the force constant $k_{\mathrm{e}}$.

A more precise analysis of relaxation processes in liquid solutions cannot be performed for the time being due to the lack of precise time resolved fluorescence spectra with a sufficiently large delays between excitation and emission. But the aim of this paper is to show what information can be gained by means of the proposed analyzing procedure. We do not intend to perform a precise analysis of a particular molecular system.

\section{Summary}

A new analytic procedure for interpretation of electronic spectra of polar dye solutions is given. A quasi-molecule model of the spectra extended to the case of time dependent fluorescence shifts was applied. It was shown that profiles of absorption and fluorescence spectra of such systems deliver several numbers of molecular parameters like energy of pure electronic transitions and relative values of effective force constants in the ground and excited states of quasi-molecules. These parameters can be useful in investigations of solvatochromic processes in biophysical, biochemical and other complex molecular systems. It has to be emphasized that the quasi-molecule concept is not a new invention. In fact it has been introduced by Jablonski in 1931 as a concept of a luminescence centre [21].

\section{References}

[1] B.I. Stepanov, V.P. Gribkovski, Theory of Luminescence, Iliffe Books, London 1968, p. 170.

[2] A. Stuart, Advanced Theory of Slatislics, Vol. 1, Oxford University Press, Oxford 1987.

[3] D.B. Siano, D.E. Metzler, J. Chem. Phys. 51, 1856 (1969).

[1] D.L. Dexter, Solid Slate Phys. 6, 390 (1958).

[5] N.G. Bakshiev, Solvatochromia, Univ, of Leningrad, 1989.

[6] A. Bączyński, P. Targowski, B. Ziętek, D. Radomska, Z. Nat.forsch. A 45, 618 (1990).

[7] A. Penzkofer, W. Falkenstein, W. Kaiscr, Chem. Phys. Lett. 44, 82 (1976); D. Reiser, A. Laubereau, Opt. Commun. 42, 329 (1982); A.M. Weiner, E.P. Ippen, Chem Phys. Lett. 114, 456 (1985).

[8] Yu.T. Mazurenko, N.G. Bakshiev, Opt. Spektrask. 28, 490 (1970).

[9] B. Bagchi, D.W. Oxtoby, G.R. Fleming, Chem. Phys. 86, 257 (1984).

[10] G. van der Zwan, J.T. Hynes, J. Phys. Chem. 89, 4181 (1985).

[11] L.A. Hallidy, M.R. Topp, J. Phys. Chem. 82, 2415 (1978).

[12] Yu.T. Mazurenko, V.S. Udaltsov, Opl. Spcklrosk. 44, 417 (1977).

[13] T. Okamura, M. Sumitami, K. Yoshihara, Chem. Phys. Lett. 94, 339 (1983).

[14] E.W. Castner, M. Marconelli, G.R. Fleming, J. Chem Phys. 88, 1090 (1987). 
[15] F. Loring, Yi Ying Yan, S. Mukamel, Chem. Phys. Lett. 135, 23 (1987).

[16] A. Mokhtari, J. Chesnoy, A. Laubereau, Chem. Phys. Lett. 155, 593 (1989).

[17] A. Bączyński, P. Targowski, B. Ziętek, D. Radomska, Z. Nat.forsch. A 45, 1349 (1990).

[18] M. Lax, J. Chem. Phys. 20, 1752 (1952).

[19] H. Mahr, M.D. Hirsch, Opt. Commun. 13, 96 (1975).

[20] A. Laubereau, A. Seilmeier, W. Kaiser, Chcm. Phys. Lett. 36, 2329 (1975).

[21] A. Jabłoński, Z. Phys. 73, 460 (1932). 